\title{
Oncology and complications
}

Giuseppe Giordano ${ }^{1}$, Evangelia Kyriazi ${ }^{2}$, Charalampos Mavridis ${ }^{3}$, Francesco Persico ${ }^{4,5}$, Charalampos Fragkoulis ${ }^{6}$, Piergiorgio Gatto ${ }^{7}$, George Georgiadis ${ }^{3}$, Irene Giagourta ${ }^{2}$, Ioannis Glykas ${ }^{6}$, Rodolfo Hurle ${ }^{4}$, Massimo Lazzeri ${ }^{4}$, Giovanni Lughezzani ${ }^{4,8}$, Vincenzo Magnano San Lio ${ }^{1}$, Charalampos Mamoulakis ${ }^{3}$, Diego Meo ${ }^{1}$, Helen A. Papadaki ${ }^{9}$, George Piaditis ${ }^{2}$, Charalampos Pontikoglou ${ }^{9}$, Georgios Stathouros ${ }^{6}$

${ }^{1}$ Unit of Diagnostic and Interventional Radiology, ARNAS "Garibaldi-Nesima”, Catania, Italy;

2 Endocrinology Department, General Hospital of Athens "G. Gennimatas", Athens, Italy;

${ }^{3}$ Department of Urology, University General Hospital of Heraklion, University of Crete, Medical School, Heraklion, Crete, Greece;

${ }^{4}$ Humanitas Clinical and Research Center - IRCCS, Department of Urology, Rozzano, Milan, Italy;

${ }^{5}$ University of Naples Federico II, Department of Neurosciences, Sciences of Reproduction, and Odontostomatology, Naples, Italy;

${ }^{6}$ Urology Department, General Hospital of Athens "G. Gennimatas", Athens, Greece;

${ }^{7}$ Ospedale di Sestri Levante - ASL 4 Liguria, Dipartimento Medico ad Elevata Integrazione Territoriale, Sestri Levante, Italy;

${ }^{8}$ Humanitas University, Department of Biomedical Sciences, Rozzano, Milan, Italy;

${ }^{9}$ Department of Hematology, University General Hospital of Heraklion, University of Crete, Medical School, Heraklion, Crete, Greece.

\section{Summary This collection of cases describes some unusual urological tumors and complica- tions related to urological tumors and their treatment.}

Case 1: A case of uretero-arterial fistula in a patient with long-term ureteral stenting for ureteral oncological stricture and a second case associated to retroperitoneal fibrosis were described. Abdominal CT, pyelography, cystoscopy were useful to show the origin of the bleeding. Angiography is useful for confirming the diagnosis and for subsequent positioning of an endovascular prosthesis which represents a safe approach with reduced post-procedural complications.

Case 2: A case of patient who suffered from interstitial pneumonitis during a cycle of intravesical BCG instillations for urothelial cancer. The patient was hospitalized for more than two weeks in a COVID ward for a suspected of COVID-19 pneumonia, but he did not show any evidence of SARS-CoV-2 infection during his hospital stay.

Case 3: A case of a young man with a functional urinary bladder paraganglioma who was successfully managed with complete removal of the tumor, leaving the urinary bladder intact.

Case 4: A case of a 61 year old male suffering from muscle invasive bladder cancer who was admitted for a radical cystectomy and on the eighth postoperative day developed microangiopathic hemolytic anemia and thrombocytopenia, which clinically defines thrombotic microangiopathy.

KEY WORDS: Ureter-arterial fistula; BCG; Pneumonitis; Bladder tumors; Cystectomy; Paraganglioma; Thrombotic thrombocytopenic purpura.

Submitted 26 July 2020; Accepted 21 August 2020

\section{Case 1 \\ ROLE OF INTERVENTIONAL RADIOLOGY IN THE TREATMENT OF URETERO-ARTERIAL FISTULAS: TWO-CASES OF ENDOVASCULAR TREATMENT} (Giuseppe Giordano, Diego Meo, Vincenzo Magnano San Lio )

\section{INTRODUCTION}

Uretero-arterial fistulas (FUA) are defined as a pathological connection between the ureter and the iliac artery $(1,2)$. The known risk factors for this pathology are pelvic or genitourinary surgery, pelvic radiotherapy treatments, vascular pathologies such as iliac aneurysms and being chronic carriers of ureteral stents (3). It represents a rare occurrence that is found today more frequently in relation to the increase in life expectancy of patients with pelvic neoplasms (4). It is difficult to diagnose and it is associated with high rates of morbidity and mortality. Different therapeutic approaches are possible, in particular interventional radiology techniques, ranging from the use of balloon catheters to intra-arterial embolization and placement of covered stents, have proven to be a less invasive and faster option, offering excellent results in terms of resolution of the pathology. In this report we describe, in addition to the role of interventional radiology in the treatment of this pathology, two cases successfully treated at our center with an endovascular approach.

\section{Case report}

A patient (male, 61 years old) was subjected to left hemicolectomy for neoplasia in 2017 and subsequently to pelvic exenteratio with uretero-cutaneous-ileostomy according to Bricker for loco-regional relapse. In January

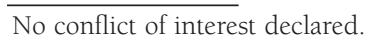


2018 in the presence of bilateral hydroureteronephrosis, due to tightened stenosis at the level of the ileal ureteral anastomosis caused by the appearance of pathological tissue attributable to recurrent disease, nephrostomies were placed with subsequent stenting of the ureters with a 8 Fr pig tail stent. One year later (January 2019) repeated episodes of intermittent hematuria occurred. In the same month, during a routine control for replacement of urinary stents, performed in the angiography room, a copious bleeding from the right mono J ureteral catheter was appreciated. Pyelography was then performed from the ureteral stent. Increasing pressure in the ureter, opacification of the common ipsilateral iliac artery was observed through reflux (Figure 1).

In the same session, therefore, through right trans-

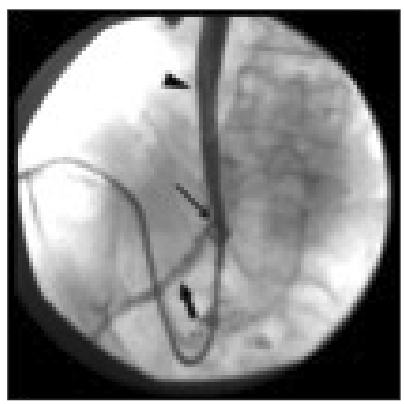

Case 1 - Figure 1. Pyelography at high pressure showing opacification of the common iliac artery.

Case 1 - Flgure 2.

Pyelography at high pressure showing opacification of the common iliac artery.
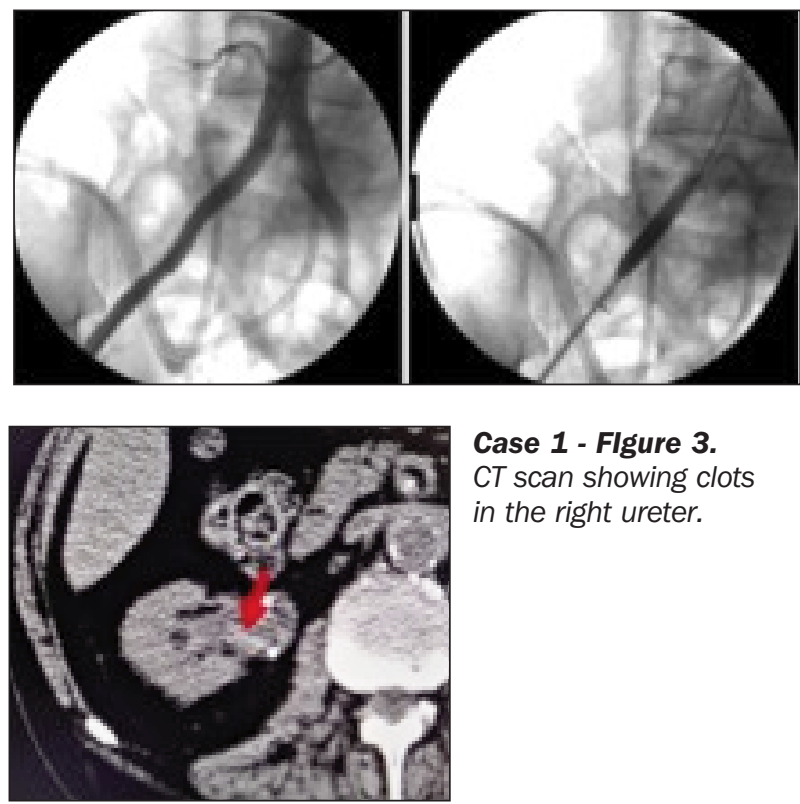

Case 1 - Flgure 3. CT scan showing clots in the right ureter.

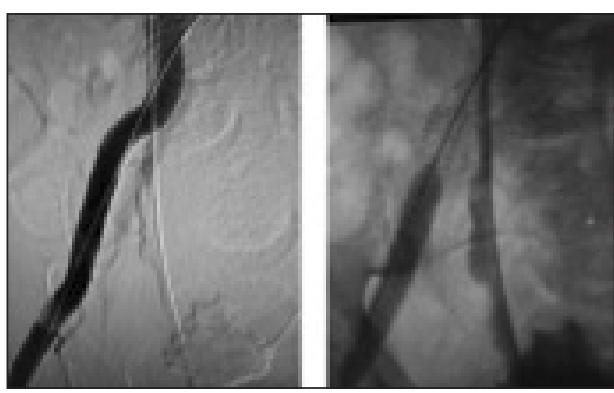

Case 1 Flgure 4. Placement of uncoated balloon expandable stent $8 \mathrm{~mm}$ diameter 60 $\mathrm{mm}$ long in the external iliac artery. femoral approach with a 9 Fr introducer, it was positioned at the level of the left common iliac artery, on a 0.035 guide, a metal coated stent $9 \mathrm{~mm}$ diameter $60 \mathrm{~mm}$ long that was subsequently dilated with a $8 \mathrm{~mm}$ diameter $4 \mathrm{~cm}$ long balloon catheter (Figure 2).

At the end of the procedure, complete stop of bleeding was observed, which no longer occurred until to the patient's death after about 6 months due to progression of the neoplastic pathology. A patient (male 63 years old) affected by retroperitoneal fibrosis determining bilateral hydroureteronephrosis was subjected to bilateral ureteral stenting with cystoscopic approach; after repeated episodes of hematuria and severe anemization with need for blood transfusions in May 2019, he performed a CT scan in June 2019 that did not show any signs of active bleeding but showed the presence of clots in the right ureter (Figure 3).

In relation to the finding, confirmed by a cystoscopic evaluation with direct visualization of clots in the bladder coming from the right ureter, the patient was taken to the angiographic room and, after recovering the bladder end of the right urinary stent with a goose neck catheter, an introducer $7 \mathrm{Fr} 35 \mathrm{~cm}$ long was advanced on a metallic guide via transurethral access to the right ureteral meatus to perform a pyelography which however does not show a fistulous route to the iliac artery. However, due to the numerous episodes of hematuria, the clots inside the right ureter, the absence of other bleeding foci and the contiguity of the right ureter with the right common iliac artery at the passage in the external iliac, it was decided to position a $10 \mathrm{~mm}$ diameter 59 $\mathrm{mm}$ long modular stent via a right transfemoral approach using an $8 \mathrm{Fr}$ introducer and to dilate it in its proximal portion in the common iliac with a $12 \mathrm{~mm}$ diameter 40 $\mathrm{mm}$ long balloon catheter.

The procedure was completed by placing an additional uncoated balloon expandable stent $8 \mathrm{~mm}$ diameter 60 $\mathrm{mm}$ long in the external iliac artery in the presence of a iatrogenic focal dissection (Figure 4).

The patient, currently was undergoing follow-up and no longer presented hematuria with resolution of anemia.

\section{Conclusions}

Despite the paucity of the literature data, due also to the rarity of this pathology, the interventional radiology techniques, compared to surgery, should be considered as first choice, especially in urgency, for treatment of uretero-arterial fistula; in particular the positioning of covered stents at the level of the artery in correspondence with the fistula has proved to be a fast, safe and effective system in resolving the hemorrhagic situation and increasing the survival of these patients. Thanks also to technological upgrade of modular coated stents, today it is possible to treat fistulous lesions by more precisely calibrating the dimensions of the prosthesis even in vascular districts with different diameters, obtaining a greater hold of the stent with the vessel walls.

\section{References}

1. Moschowitz A. Simultaneous ligation of both external iliac arteries for secondary hemorrhage following bilateral ureterolithotomy. Ann Surg. 1908; 48:872-5. 
2. Van den Bergh RC, Moll FL, de Vries JP, et al. Arterioureteral fistulas: unusual suspects systematic review of 139 cases. Urology. 2009; 74:251-5.

3. Krambeck AE, DiMarco DS, Gettman MT, et al. Ureteroiliac artery fistula: diagnosis and treatment algorithm. Urology. 2005; 66:990-4.

4. Fox JA, Krambeck A, McPhail EF, et al. Ureteroarterial fistula treatment with open surgery versus endovascular management: long-term outcomes. J Urol. 2011; 185:945-50.

\section{Case 2 \\ The effect of Bacillus Calmette-Guérin (BCG) INSTILLATIONS ON THE IMMUNE SYSTEM: A CASE REPORT OF HYPERSENSITIVITY PNEUMONITIS DURING THE COVID-19 PANDEMIC}

(Francesco Persico, Massimo Lazzeri, Giovanni Lughezzani, Piergiorgio Gatto, Rodolfo Hurle)

\section{INTRODUCTION}

BCG is a live attenuated vaccine derived from Mycobacterium bovis that was developed against tuberculosis at the beginning of the $20^{\text {th }}$ century at the Institut Pasteur in Paris (1). Since then, it has been the most used vaccine in the world, with around 130 million children vaccinated every year. Moreover, BCG intravesical instillation is a widely used treatment for high-risk non-muscle invasive Bladder Cancer (NMIBC) (2).

Although the exact mechanism of BCG on tumor prevention is still unclear, a local immune response is presumed. The BCG-induced activation of the immune system involves CD4(+) and CD8(+) lymphocytes, natural killer cells, macrophages, granulocytes, and dendritic cells. Bladder cancer cells are killed through cell-mediated cytotoxicity, by the secretion of soluble factors such as TRAIL (tumour necrosis factor-related apoptosis-inducing ligand), and, to a certain extent, by the direct action of BCG (3). Recent studies has also suggested that the trained immunity induced by BCG vaccination is protective against multiple infections (1).

In this case report, we presented a peculiar case of a male patient with a medical history of recent BCG instillations for NMIBC who suffered from interstitial pneumonitis and did not contract SARS-CoV-2 infection despite his prolonged hospitalization in a COVID ward.

\section{Case report}

A 63-year-old man was admitted to the ER for the suspect of a SARS-CoV-2 induced interstitial pneumonia during BCG treatment for high-grade NIMBC. He did not have a personal history of direct exposure to individuals with SARS-CoV-2 infection.

The patient's past medical history included a diagnosis of high-grade NMIBC in 2012 followed by a BCG induction cycle (one instillation weekly for 6 weeks) and maintenance (one instillation for 3 weeks on months 3, 6, 12, $18,24)$ [SWOG protocol] in 2012-2013.

During follow-up, in July 2017, he underwent a diagnostic ureteroscopy for suspicion of upper tract urothelial neoplasm based on positive urinary cytology and a suspicious computed tomography (CT). Biopsies histological examination revealed a carcinoma in situ (CIS) of left ureter. Consequently, the patient underwent an open left nephroureterectomy for CIS of the left ureter in September 2017. After surgery, he resumed BCG instillations and underwent 3 bladder mappings resulted negative for urothelial cancer. During the treatment, the patient developed severe irritative symptoms, suggestive for BCG-induced chemical cystitis, that were treated first conservatively with hyaluronic acid instillations in April 2019. In January 2020, after a negative cystoscopy with suspicious cytology, he underwent right flexible ureterorenoscopy with mapping of renal pelvis (negative for urothelial cancer) and selective urinary cytology of right upper urinary tract (suspicious for malignancy). During the procedure, the surgeon placed a JJ ureteral stent 6 French x 28 Centimeters to perform upper urinary tract instillations. The patient subsequently received intravesical Bacillus Calmette-Guerin (BCG) instillation at weekly intervals. Four doses were administered without relevant problems. After the fourth BCG instillation on 13th of March, the patient developed influenza-like symptoms; fever, anorexia, night sweats, shivering, cough, and chest distress persisted for about three weeks, with the body temperature reaching a maximum of $38.1^{\circ} \mathrm{C}$. On March $27^{\text {st }}$, the ureteral stent was removed for a suspicion of an urinary infection and the patient started an empirical antibiotic therapy with ciprofloxacin and trimetoprim-sulfametoxazole as prescribed by his general practicioner.

On March $31^{\text {st }}$, he came to the emergency department of the Hospital of Sestri Levante for a respiratory distress and a physical examination highlighted fine inspiratory crackles in both lungs. Upon admission, the patient's body temperature was $38.3^{\circ} \mathrm{C}$. Haemogasanalysis showed oxygen pressure $\left(\mathrm{PO}_{2}\right)$ and Carbon Dioxide pressure $\left(\mathrm{PCO}_{2}\right)$ of $43 \mathrm{mmHg}$ and $30 \mathrm{mmHg}$ on room air, respectively, with $\mathrm{pH}$ of 7.5 . The blood exams showed leukocytosis (16760 cells $/ \mathrm{mm}^{3}$ ) with increased levels of C-reactive protein $(6 \mathrm{mg} / \mathrm{dL})$ D-Dimer, transaminases, LDH and creatinine $(1.54 \mathrm{mg} / \mathrm{dL})$. Chest radiography highlighted widespread accentuation of the bronchovascular texture. Therefore, he was admitted to a COVID ward with the suspicion for COVID-19 pneumonia. According to institutional protocols, he began a therapy with clarithromycin, ceftriaxone, hydroxychloroquine, enoxaparin and oxygen therapy. The patient performed on the $1^{\text {st }}$ and on the $11^{\text {th }}$ of April two SARS-CoV-2 oropharyngeal swabs that resulted negative. Serology for SARS-CoV-2 was negative as well. Blood culture and urine culture performed on the April $1^{\text {st }}$ were both negative. On April $8^{\text {th }}$, a chest CT was performed revealing a bilateral subpleural patchy ground-glass appearance associated to the presence of small centrilobular nodules (Figure 1). On April $10^{\text {th }}$, the patient underwent bronchoscopy with Broncho-Alveolar Lavage (BAL) that resulted negative for BCG and SARS-CoV-2. The clinical status improved gradually with normalization of PCR, DDimer, transaminases, LDH and creatinine. Oxygen therapy was suspended on April $13^{\text {th }}$ and the patient was discharged on April $15^{\text {th }}$ with diagnosis of hypersensitivity pneumonitis BCG-induced. Medical therapy consisted of omeprazole, enoxaparin, levofloxacin and prednisone for one month. On June $22^{\text {th }}$ the patient underwent uri- 


\section{Case 2 - Flgure 1.}

Chest imaging of the patient.

The computed tomography scans taken on the $8^{\text {th }}$ of April 2020 showed multiple patchy ground-glass opacities in bilateral subpleural areas with small nodular formations with centerlobulary localization. The image is published under agreement of the patient.

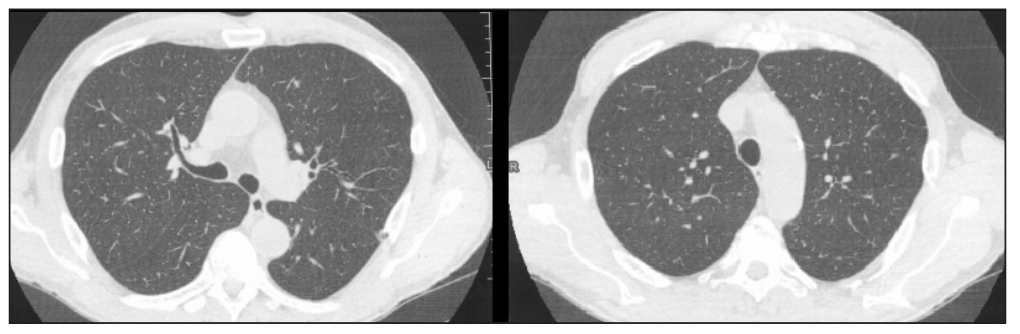

nary cytology and transurethral cystoscopy that were both negative. He did not report significative respiratory symptoms and abnormal findings on chest CT disappeared.

\section{Conclusions}

This case report emphasizes the importance of understanding the real effect of BCG on immune system. Further researches are needed to understand the possible protective role of immunotherapy with BCG in presence of COVID-19 infection.

\section{References}

1. Arts RJW, et al. BCG Vaccination Protects against Experimental Viral Infection in Humans through the Induction of Cytokines Associated with Trained Immunity. Cell Host Microbe. 2018; 23:89100.e5.

2. Malmstrom PU, et al. An individual patient data meta-analysis of the long-term outcome of randomised studies comparing intravesical mitomycin C versus bacillus Calmette-Guerin for nonmuscleinvasive bladder cancer. Eur Urol. 2009; 56:247.

3. Redelman-Sidi G, Glickman MS, Bochner BH. The mechanism of action of BCG therapy for bladder cancer-a current perspective. Nat Rev Urol. 2014; 11:153-162.

\section{CASe 3}

Paraganglioma of the URINARY BLAdDer.

A CASE REPORT AND REVIEW OF THE LITERATURE (Evangelia Kyriazi, Charalampos Fragkoulis, Irene Giagourta, Ioannis Glykas, Georgios Stathouros, George Piaditis)

Tumors arising from the chromaffin cells, derived from the embryonic neural crest, usually originate from the adrenal medulla, are designated as pheochromocytomas. Paragangliomas (PGLs) are such tumors occurring in extra-adrenal locations and represent $10 \%$ of all pheochromocytomas (1). PLGs are either functional or non-functional depending in the secretion of catecholamines (2). Urinary bladder PLGs are rare as they represent $6 \%$ of all PLGs with most common locations the dome or the trigone. PLGs located in other sites of the genitourinary system are less common as bladder is the primary site of such tumors $(80 \%)$ followed by the urethra $(12.7 \%)$, pelvis $(4.9 \%)$ and ureter $(3.2 \%)(3.4)$.
In the current case-report, we present a young man with a functional urinary bladder paraganglioma who was successfully managed with complete removal of the tumor, leaving the urinary bladder intact.

\section{Case report}

A 38-year-old man experiencing palpitation, headache, diaphoresis, and abdominal painafter micturition was referred to the endocrinology Department for evaluation and further investigation. When further enquired, he described an 8 year history of the aforementioned symptoms, accompanied by characteristic facial pallor and high blood pressure ranging from 170/95 $\mathrm{mmHg}$ to 190/110 mmHg. Apart from the moment of episodes coincided with urination, the patient's blood pressure was marginally elevated. His physical examination and medical history were otherwise unremarkable.

The clinical suspicion of a PHEO/PGL was confirmed by a 24h urine collection demonstrating elevated levels of urine metanephrine $3257(100-800 \mu \mathrm{g} / 24 \mathrm{~h})$ and normetanephrine 3177 (88-44 $\mu \mathrm{g} / 24 \mathrm{~h})$. Subsequently, a CT scanning of the abdomen documented the presence of a multi-lobular, homogenously contrast-enhanced mass $(5.5 \mathrm{~cm} \times 3.5 \mathrm{~cm})$ arising from the right posterior wall of the urinary bladder. A CT scanning of his neck and thorax was performed but did not reveal any additional pathological findings.

Patient was referred to the Urology Department to surgically remove the mass. Preoperative preparation included the administration of phenoxybenzamine (a-adrenergic blocking agent) and fluids ( $\mathrm{NaCl} 0.9 \%)$ in order to restore arterial blood pressure and intravascular volume and avoid intra-operative hypertensive crisis and postresection hypotension. A sub-umbilical midline incision was performed, bladder was mobilized, right ureter was recognized, and the mass was resected from the bladder wall detrusor muscle. The resection was successfully performed without opening the bladder. A single extraperitoneal drain was placed which was removed on the third postoperative day. Blood loss during surgery was minimal $(<100 \mathrm{ml})$. No complications were recorded postoperatively. Patient remained normotensive and was subsequently discharged without receiving any antihypertensive treatment.

Pathology report confirmed the presence of a PGL embedded in smooth muscle fibers of the urinary bladder detrusor muscle. Macroscopically, the tumor was a solid, ovular, brown in color mass $(4.5 \times 3.5 \times 3 \mathrm{~cm})$ which was partially encapsulated. Microscopically, the neoplasm had developed among smooth muscle cells, was of moderate to high cellularity and had grown in a typical "zellbalen" pattern. The cells had abundant, amphophilic cytoplasm with moderate to high nuclear pleomorphism. Focally located giant multinucleated cells were also observed. Mitoses were scarce (0-1/10 High Power Fields). Neither necrosis nor vascular invasion was observed. The tumor cells were positive for CgA, SYP, NSE, focally positive for S-100 and p53 (1$2 \%$ ) and negative for CKAE1/AE3, p63, Her2-neu. 
The proliferation index Ki67 (MIB-1) was estimated > 3\% (focally up to $8 \%$ ). Follow-up protocol included a $24 \mathrm{~h}$ urine collection for metanephrines 3 months after surgery which was normal and a post-operative MRI of the abdomen which revealed no pathological findings. Our patient had neither clinical features implying a syndrome related to PGL nor a positive family history. Nevertheless, taking into consideration his young age and the histopathologically proven presence of PGL, we proceeded to his genetic testing to exclude a germline mutation. No disease-associated sequence changes were identified in SDHB, SDHC, SDHD, SDHA and SDHAF2 genes.

\section{Conclusions}

Paraganglioma of the urinary bladder is a rare tumor which, when secretory, has a typical presentation usually related to micturition. In our case, owing to the tumor's favorable location, the patient was managed with complete resection of his mass leaving the bladder mucosa intact and without the need of partial cystectomy. Our case is an exception to the rule, given that urinary bladder PGLs management usually entails more invasive and extensive procedures. Genetic testing should always be considered, because it provides with invaluable information which may modify our patients' surveillance and improve their prognosis.

Finally, it is worth underscoring that patients with PGLs should receive life-long follow-up because of the peculiar biological behavior and the malignant potential of their disease.

\section{REFEREnCES}

1. Leestma JE, Price EB Jr. Paraganglioma of the urinary bladder. Cancer. 1971; 28:1063-73.

2. Al-Zahrani AA. Recurrent urinary bladder paraganglioma. Adv Urol. 2010; 2010:912125.

3. Peng C, Bu S, Xiong S, et al. Non-functioning paraganglioma occurring in the urinary bladder: A case report and review of the literature. Oncol Lett. 2015; 10: 321-324.

4. Lenders JW, Duh QY, Eisenhofer G, et al. Endocrine Society. Pheochromocytoma and paraganglioma: an endocrine society clinical practice guideline. J Clin Endocrinol Metab. 2014; 99:1915-42.

\section{Case 4}

THROMBOTIC THROMBOCYTOPENIC PURPURA AS A COMPLICATION AFTER RADICAL CYSTECTOMY (Charalampos Mavridis, George Georgiadis, Charalampos Pontikoglou, Helen A. Papadaki, Charalampos Mamoulakis)

\section{INTRODUCTION}

Thrombotic Thrombocytopenic Purpura (TTP) is a rare entity associated with Microangiopathic Hemolytic Anemia (MAHA) and thrombocytopenia (1). Acute kidney injury, fever, and neurological symptoms may also be present. TTP is fatal if left untreated. Triggering factors usually remain unknown but major surgery-induced stress may result in TTP (1). The aim of the present report is to highlight the importance of close follow-up during the postoperative period following major surgery and to underline the importance of immediate PEX treatment. To the best of our knowledge, this is the first TTP case reported after radical cystectomy.

\section{Case report}

A 61 year old male with multiple high grade/in situ transitional cell bladder tumor and squamous cell bladder cancer and with a past medical history significant for hypertension and diabetes mellitus was admitted for elective radical cystectomy. Abdominal/thorax computed tomography (CT) scans and bone scintigraphy showed no metastases and the preoperative laboratory work-up was insignificant as well. The perioperative course was uneventful; two units of red blood cells (RBC) were intraoperatively transfused. On the $6^{\text {th }}$ post-operative day (PD), there was an increase in serum LDH level and a decrease in PLT count. PLT count decrease was initially attributed to the low-molecular-weight heparin administration (heparin-induced thrombocytopenia), which was then replaced by fondaparinux. On the $8^{\text {th }} \mathrm{PD}$ he developed uncontrolled hyperglycemia, confusion, headache and blurred vision. Arterial blood gas levels were normal and brain, abdomen-pelvis CT scans showed no abnormalities. Laboratory tests revealed an acute HT and PLT count decrease, negative direct Coombs test, normal prothrombin/activated partial thromboplastin time, normal fibrinogen level, normal troponin level and mildly elevated ddimers. Peripheral blood smear revealed five schistocytes per high power field, thereby defining microangiopathic anemia. The combination of microangiopathic anemiathrombocytopenia suggested thrombotic microangiopathy (TMA). A second sample sent for analysis within hours showed further HT and PLT count deterioration, acute increase in LDH and acute kidney injury (Table 1).

In view of these findings and taking into consideration

Case 4 - Table 1.

Laboratory results and PEX procedures.

\begin{tabular}{|c|c|c|c|c|c|c|c|}
\hline & $\begin{array}{l}\text { Crea } \\
(\mathrm{mg} / \mathrm{dl})\end{array}$ & $\begin{array}{c}\text { Thil } \\
\text { (mg/dl) }\end{array}$ & $\begin{array}{c}\text { WBC } \\
\text { count } \\
(103 / \mu \mathrm{l})\end{array}$ & $\begin{array}{c}\text { PLT } \\
\text { count } \\
\text { (109/L) }\end{array}$ & $\begin{array}{l}\text { Ht } \\
(\%)\end{array}$ & $\begin{array}{l}\text { LDH } \\
\text { (IU/L) }\end{array}$ & $\begin{array}{c}\text { Schistocytes } \\
\text { (per HPF) }\end{array}$ \\
\hline$\overline{D O S}$ & 1.04 & 1.01 & 11.7 & 242 & 36 & 360 & n.a. \\
\hline PD1 & 1.07 & 2.6 & 12.7 & 251 & 35.1 & 390 & ก.a. \\
\hline$\overline{\mathrm{PD} 2}$ & 1.12 & 2.3 & 14.5 & 271 & 35.2 & 410 & n.a. \\
\hline PD3 & 1.19 & 1.29 & 11.6 & 333 & 33 & 382 & n.a. \\
\hline PD4 & 0.9 & 0.9 & 14.2 & 277 & 34.1 & 390 & n.a. \\
\hline$\overline{\mathrm{PD} 5}$ & 1.08 & 1.4 & 16.9 & 228 & 34.6 & 380 & n.a. \\
\hline$\overline{P D 6}$ & 1.06 & 1.9 & 15 & 110 & 33.6 & 525 & n.a. \\
\hline$\overline{\mathrm{PD7}}$ & 1.11 & 1.8 & 14 & 35 & 30.6 & 705 & n.a. \\
\hline$\overline{P D 8}$ & 1.47 & 2.8 & 20 & 8 & 21 & 1024 & 5 \\
\hline PD9 $\left(1^{\text {st PEX })}\right.$ & 1.55 & 3.6 & 27.1 & 19 & 17.2 & 1246 & 3 \\
\hline PD10 (2n PEX) & 1.39 & 4.4 & 22.9 & 41 & 23.8 & 1705 & 2 \\
\hline PD11 (3 PEX) & 1.35 & 3.2 & 32.5 & 59 & 26 & 707 & n.a. \\
\hline$\overline{P D 12\left(4^{\text {th }} \text { PEX) }\right.}$ & 1.27 & 1.85 & 36.1 & 88 & 27 & 622 & 1 \\
\hline$\overline{P D 13}\left(5^{\text {th }}\right.$ PEX) & 1.25 & 1.35 & 34 & 84 & 28.9 & 540 & 1 \\
\hline$\overline{P D 14\left(6^{\text {th }} \text { PEX }\right)}$ & 1.22 & 1.35 & 14.5 & 140 & 30 & 440 & n.a. \\
\hline$\overline{\mathrm{PD} 15}$ & 1.09 & 0.9 & 23 & 170 & 30.1 & 300 & n.a. \\
\hline PD16 & 1.09 & 0.8 & 15.1 & 204 & 30.4 & 175 & 0 \\
\hline$\overline{P D 33}$ & 1.02 & 0.5 & 10.9 & 290 & 33.2 & 221 & n.a. \\
\hline
\end{tabular}


the neurological symptoms of the patient the presumptive diagnosis of TTP was made and immediate treatment was initiated: prednisolone ( $25 \mathrm{mg}$ t.i.d.) intravenously plus transfusion of two RBC units and nine units of fresh frozen plasma as a temporary measure since PEX therapy, the mainstay treatment of TTP (2), is not readily available in our hospital. The next day (9 $\left.{ }^{\text {th }} \mathrm{PD}\right)$, PEX therapy was initiated. The patient eventually improved and was discharged on the $33^{\text {st }} \mathrm{PD}$ in good condition. Later the diagnosis was made by adamts 13 activity test.

\section{Conclusions}

Major surgery in a patient with coexisting chronic and severe disorders may trigger TTP due to release of unknown factors that can cause disruption of homeostatic blood mechanisms, thus it is deemed crucial to closely follow-up these patients even beyond the $7^{\text {th }}$ PD. TTP should be considered in the differential diagnosis of MHA and thrombo- cytopenia, associated with renal impairment and neurological manifesta-tions that occurs during the postoperative period, in the absence of another clinically apparent etiology. Since TTP is fatal if left untreated, physicians must be aware of this rare postoperative complication and immediate PEX therapy should be initiated.

\section{ACKNOWLEDGEMENTS}

The authors would like to thank the Special Research Account of the University of Crete for supporting the publication of this study (ELKE No 3550).

\section{References}

1. Eskazan AE, Buyuktas D, Soysal T. Postoperative thrombotic thrombocytopenic purpura. Surg Today. 2015; 45:8-16.

2. Scully M, Goodship T. How I treat thrombotic thrombocytopenic purpura and atypical haemolytic uraemic syndrome. Br J Haematol. 2014; 164:759-66.

\section{Correspondence}

Giuseppe Giordano, MD

g.giorda@gmail.com

Diego Meo, MD (Corresponding Author)

diegomeo@hotmail.it

Vincenzo Magnano San Lio, MD

v.magnano@alice.it

Unit of Diagnostic and Interventional Radiology ARNAS "Garibaldi-Nesima"

Via Palermo 636, 95122 Catania (Italy)

Francesco Persico, MD (Corresponding Author)

francesco.persico90@gmail.com

Massimo Lazzeri, MD

lazzeri.maximus@gmail.com

Giovanni Lughezzani, MD

g.lughezzani@gmail.com

Piergiorgio Gatto, $M D$

piergiorgio.gatto@asl4.liguria.it

Rodolfo Hurle, MD

rodolfo.hurle@humanitas.it

Humanitas Clinical and Research Center - IRCCS - Via Manzoni 56, 20089 Rozzano (MI) (Italy)

Evangelia Kyriazi, MD

kyrevan@windowslive.com

Charalampos Fragkoulis, MD

harisfrag@yahoo.gr

Irene Giagourta, MD

irene_giag@yahoo.com

Ioannis Glykas, MD (Corresponding Author)

giannis.glykas@gmail.com

Georgios Stathouros, MD

gstathouros@yahoo.gr

George Piaditis, MD

edk-pgna@otenet.gr

General Hospital of Athens G. Gennimatas, Athens (Greece)

Charalampos Mavridis, MD

ch.mavridis@uoc.gr

George Georgiadis, MD

geokosgeo@yahoo.gr

Charalampos Pontikoglou, MD

xpontik@uoc.gr

Helen A. Papadaki, MD

e.papadaki@uoc.gr

Charalampos Mamoulakis, MD (Corresponding Author)

mamoulak@uoc.gr

University General Hospital of Heraklion, University of Crete, Medical School, Heraklion, Crete (Greece) 